\title{
Management of skeletal-related events in patients with advanced prostate cancer and bone metastases: Incorporating new agents into clinical practice
}

\author{
Alan So, MD, FRCSC;; Joseph Chin, 0.Ont, MD, FRCSC; ${ }^{+}$Neil Fleshner, MD, FRCSC; Fred Saad, MD, FRCSC \\ *Department of Urological Sciences, University of British Columbia, Vancouver, BC; †University of Western Ontario, London Health Sciences Centre, London, ON; §Division of Urology, Department of Surgical \\ Oncology, University Health Network (UHN), University of Toronto, Toronto, ON; : University of Montreal Hospital Centre, CRCHUM, Montreal, QC
}

Cite as: Can Urol Assoc J 2012;6(6):465-70. hittp://dx.doi.org/10.5489/cuaj.12149

\section{Abstract}

Skeletal-related events (SREs) are a common complication of bone metastases, and have serious negative consequences for patients with castrate-resistant prostate cancer (CRPC). SREs can lead to severe pain, increased risk of death, increased health care costs and reduced quality of life. Until recently, zoledronic acid has been the sole standard of care for the prevention of SREs in men with CRPC with bone metastases. Denosumab, a receptor activator of nuclear factor kappa-B ligand (RANK-L) inhibitor, has been recently approved for use in Canada for this indication, thus presenting another option for these patients. Denosumab was shown to be superior to zoledronic acid in delaying the time to first or subsequent SREs in CRPC patients with bone metastases. This review discusses current and previous trials examining agents designed to prevent SREs in men with CRPC and bone metastases. It also discusses the practical aspects of administering a bone-targeted therapy, including choosing a bone-targeted therapy, monitoring at the onset and during therapy, switching from one therapy to another, and assessing potential complications.

\section{Introduction}

In Canada, prostate cancer accounts for $27 \%$ of newly diagnosed cancers in men, with an incidence of 25500 in $2011 .^{1}$ Up to $90 \%$ of men dying from prostate cancer will have bone metastases, ${ }^{2-4}$ which can be associated with skeletal-related events (SREs). SREs are defined as: a pathologic fracture, spinal cord compression, necessity for radiation to bone (for pain or impending fracture) or surgery to bone. ${ }^{5}$ Until recently, intravenous (IV) zoledronic acid was the only treatment approved in Canada for reducing the risk of SREs in CRPC patients with bone metastases. This article reviews the currently available treatments for the prevention of SREs in light of recent therapeutic advances and Canadian approvals.

\section{Bone metastases and the risk of SREs}

Metastasis to bone disrupts skeletal homeostasis by upsetting the balance between osteoblastic bone formation and osteoclast-mediated bone destruction. ${ }^{6}$ Bone metastases can lead to several potential complications, including hypercalcemia of malignancy, bone marrow failure/leukoerythroblastic anemia, and more commonly, SREs. ${ }^{7}$ If untreated, about half of advanced prostate cancer patients with bone metastases will experience at least one SRE over a 2-year period. ${ }^{8}$ The presence of three or more lesions also increases a patient's risk of SREs; ${ }^{9}$ and once a patient experiences an SRE, the risk of a subsequent SRE is increased. ${ }^{8}$ Additional insight regarding which patients are at highest risk for SREs can be provided by examining bone turnover markers, ${ }^{10}$ as altered skeletal metabolism increases levels of bone-specific biochemical markers in plasma and urine. ${ }^{11}$ Prostate cancer patients with elevated baseline and on-study levels of urinary N-telopeptide (NTx) also have a 1.57-fold and a greater than 3-fold increased risk of SREs, respectively. ${ }^{10}$ Similarly, baseline and on-study elevations of serum bone-specific alkaline phosphatase (BALP) resulted in a 1.85-fold and greater than 3-fold increased risk of SREs. ${ }^{10}$

\section{Health and economic implications of SREs}

SREs pose a significant health and economic burden. ${ }^{12,13}$ When examining quality of life parameters, such as physical well-being (e.g., pain, fatigue), emotional well-being (e.g., depression, anxiety), and functional well-being (e.g., mobility, independence), patients reported clinically significant decreases in each of these parameters after their first SRE. ${ }^{12}$ Although pain associated with SREs is difficult to study unless pain assessments are done very frequently, available data show that significant bone pain is associated with SREs; significantly fewer patients who received IV bisphosphonate therapy (zoledronic acid) to prevent SREs reported clinically significant pain requiring radiation com- 
So et al.

pared with those receiving placebo. ${ }^{14}$ The effective management of pain associated with SREs is integral to maintaining mobility and physical independence, as well as for successful treatment outcomes. ${ }^{7,15}$

Pathologic fractures have been associated with an increased risk of death in patients with prostate cancer, breast cancer or multiple myeloma. ${ }^{16}$ Prostate cancer patients on androgen- deprivation therapy are also at an increased risk of fractures. In these patients, median overall survival was significantly decreased for those with fractures compared with those without fractures. ${ }^{17}$

Moreover, osteoporotic fractures and hip fractures in the elderly population (to which most advanced prostate cancer patients belong) significantly reduce life expectancy. ${ }^{18,19}$ Together, these results demonstrate that fractures may significantly reduce survival of patients with prostate cancer. However, current treatments for preventing SREs have not been shown to improve the overall survival of these patients. ${ }^{14,20}$

In addition to serious effects on health and quality of life, SREs also have important economic consequences. ${ }^{13}$ Orthopedic or spinal surgery and other treatments for SREs add to the already significant cost burden of advanced cancer. Although there is no recent prostate cancer- specific data on the cost burden on SREs in Canada, data from other types of cancer can provide some insight. Analysis from 1999 examining the use of pamidronate in breast cancer patients showed that bone-targeted bisphosphonate treatment is cost-effective and recommended that patients receive such treatment due to the ability to delay onset of SREs and palliate the associated bone pain. ${ }^{21}$

\section{Current bone-targeted therapies}

The current treatment options for preventing or delaying SREs in patients with prostate cancer and bone metastases in Canada are zoledronic acid (Zometa, Novartis Pharmaceuticals Canada Inc.), a bisphosphonate, and denosumab (XGEVA, Amgen Canada Inc.), a RANKL inhibitor. ${ }^{22-24}$ Several other treatments (clodronate and pamidronate) have been tested in phase III clinical trials; however, only denosumab and zoledronic acid have been shown to prevent or delay SREs in patients with advanced prostate cancer and bone metastases (Table 1).

\section{Bisphosphonates}

Zoledronic acid is the only bisphosphonate shown to prevent SREs in CRPC with bone metastases. In a prospective, international, double-blind, randomized, placebo-controlled phase III trial (Zometa 039),,$^{14}$ treatment with IV zoledronic acid every 3 weeks reduced SREs and skeletal morbidities (Table 1). The trial began with two dose cohorts (4 mg and
$8 \mathrm{mg})$; two protocol modifications were made early in the trial due to concerns of renal toxicity: (1) the infusion time was lengthened from 5 to 15 minutes; and (2) the 8-mg group was reduced to $4 \mathrm{mg}$ (referred to as the 8/4 mg group). Patients receiving zoledronic acid (4 mg IV) had fewer SREs compared with patients in the placebo group $(33.2 \% \mathrm{vs}$. $44.2 \%$, respectively; $p=0.021)$. No significant reduction in the rate of SREs was seen in the $8 / 4 \mathrm{mg}$ group compared with placebo; however, this group was complicated by a high discontinuation rate. ${ }^{14}$ It is important to note that the lack of dose-dependency does not negate the efficacy observed by zoledronic acid in the 4-mg group. At the 24-month follow-up, the median time to first SRE was lengthened from 321 to 488 days for the 4-mg group ( $p=0.0009)$ and the risk of an SRE was reduced by $36 \%$ compared with placebo $(p=0.002) .{ }^{8}$ There was no statistically significant improvement in overall survival. ${ }^{8}$ The most common drug-related adverse events were fatigue, anemia, myalgia, fever and lower-limb edema. ${ }^{14}$

Renal complications were the most serious adverse event of treatment, although these were minimized by the trial modifications stated previously to the extent that no difference was observed when compared to placebo. The results of this trial led to the approval of zoledronic acid for use in patients with CRPC in Canada in 2002. ${ }^{25}$ Finally, although numerically higher, scores related to quality of life and analgesic were not significantly improved in the zoledronic acid group compared with the placebo group. In post-hoc analysis comparing patients with and without pain at baseline, the administration of zoledronic acid before the onset of bone pain appeared to be more efficacious and significantly reduced pain scores when compared with placebo. ${ }^{26}$ Thus, it is possible that zoledronic acid could have a greater benefit in certain patient subgroups.

\section{RANKL Inhibition}

Denosumab is a fully human monoclonal antibody that binds to RANKL, preventing it from binding to the RANK receptor, and therefore inhibiting osteoclastogenesis and subsequent bone resorption. An international, double-blind, phase III clinical trial examining the ability of denosumab versus zoledronic acid to delay the time to the first on-study SRE was recently published. In this event-driven trial, patients were given $120 \mathrm{mg}$ of subcutaneous (SC) denosumab and IV placebo or SC placebo and $4 \mathrm{mg}$ IV zoledronic acid every 4 weeks. The results showed that denosumab was superior to zoledronic acid in delaying the median time to first on-study SRE by 3.6 (hazard ratio [HR] 0.82, 95\% confidence interval [Cl] $0.71-0.95 ; p=0.0002) .{ }^{24}$ The absolute difference in the rate of SREs (total confirmed events) between the two groups was $5 \%(41 \%$ in zoledronic acid group and $36 \%$ in the denosumab group). The frequency of drug-related adverse 


\begin{tabular}{|c|c|c|c|c|}
\hline Study & $\begin{array}{c}\text { Study design and patient } \\
\text { population }\end{array}$ & Treatment arms (N) & Primary endpoint & Results \\
\hline MRC Pr05 ${ }^{41}$ & $\begin{array}{c}\text { Randomized, double-blind, } \\
\text { placebo-controlled; androgen } \\
\text { sensitive with bone metastases }\end{array}$ & $\begin{array}{c}\text { Daily oral } \\
\text { clodronate } \\
\text { versus placebo (311) }\end{array}$ & Symptomatic PFS & $\begin{array}{l}\text { Trend towards bone progression- } \\
\text { free survival }(p=0.066)\end{array}$ \\
\hline Study 032/INT ${ }^{42}$ & $\begin{array}{l}\text { Two-centre, randomized, } \\
\text { placebo-controlled; CRPC }\end{array}$ & $\begin{array}{l}\text { Pamidronate vs. } \\
\text { placebo, every } 3 \\
\text { weeks (350) }\end{array}$ & $\begin{array}{c}\text { Self-reported pain score, } \\
\text { analgesic use, proportion } \\
\text { of patients with SRE }\end{array}$ & $\begin{array}{l}\text { No significant difference between } \\
\text { treatment arms }\end{array}$ \\
\hline Zometa $039^{8,14}$ & $\begin{array}{l}\text { Randomized, double-blind, } \\
\text { placebo-controlled; CRPC with } \\
\text { bone metastases. }\end{array}$ & $\begin{array}{l}\text { IV zoledronic acid } \\
4 \text { mg and } 8 \text { mg vs. } \\
\text { placebo, every } 4 \\
\text { weeks (643) }\end{array}$ & $\begin{array}{c}\text { Proportion of patients } \\
\text { who experienced at least } \\
\text { one SRE }\end{array}$ & $\begin{array}{l}\text { Significant decrease in SREs } \\
\text { in zoledronic acid group at } 15 \\
\text { months ( } 44.2 \text { vs. } 33.2 \% ; p=0.021 \text { ); } \\
\text { trend towards improved survival }\end{array}$ \\
\hline Denosumab $103^{24}$ & $\begin{array}{l}\text { Randomized, double-blind, } \\
\text { double-dummy; CRPC } \\
\text { with bone metastases }\end{array}$ & $\begin{array}{c}\text { Denosumab } 120 \text { SC } \\
\text { vs. zoledronic acid } \\
4 \text { mg, every } 4 \text { weeks } \\
\text { (1904) }\end{array}$ & $\begin{array}{l}\text { Time to first on-study } \\
\text { SRE for non-inferiority } \\
\text { versus zoledronic acid }\end{array}$ & $\begin{array}{l}\text { Denosumab was superior to } \\
\text { zoledronic acid in delaying median } \\
\text { time to first SRE (20.7 vs. } 17.1 \\
\text { months; } p=0.0002) .\end{array}$ \\
\hline
\end{tabular}

events was similar between denosumab and zoledronic acid, although more cases of hypocalcemia were reported with the denosumab group compared with the zoledronic acid group ( $13 \%$ vs. $6 \%$, respectively; $p<0.0001)$. Most cases of hypocalcemia were asymptomatic; calcium decreases of grade 3 or 4 were detected in $5 \%$ of patients receiving denosumab and $1 \%$ of patients received zoledonic acid. The presence of osteonecrosis of the jaw $(\mathrm{ONJ})$ was monitored every 6 months and suspected cases were reviewed by a blinded, independent adjudicator. More patients in the denosumab arm $(22[2 \%])$ compared with the zoledronic acid arm (12 [1\%]) experienced ONJ, but the difference was not statistically significant $(p=0.09)$. Overall survival was similar between the two treatment groups. These results, as well as results from similarly designed phase III clinical trials in breast cancer and other solid tumours, led to the approval of denosumab for reducing the risk of SREs in patients with breast cancer, prostate cancer, non-small cell lung cancer and other solid tumours by Health Canada in May 2011.22 Both agents are effective in delaying onset of SREs; the small, yet significant, difference between these two agents may not be clinically relevant.

\section{Emerging bone-targeted therapies}

Radium-223 (alpharadin) is an alpha-radiation-emitting agent that specifically targets bone metastases due to its similar chemical and physical properties to calcium. It has been shown to inhibit osteoclast differentiation in vitro and in mouse models of bone metastasis. ${ }^{27}$ The ALSYMPCA trial (or the ALpharadin in SYMptomatic Prostate CAncer trial) is a phase III, randomized, double-blind, placebo-controlled, international trial examining radium-223 in patients with CRPC with bony metastases. Results from the interim analysis showed that in addition to prolonging overall survival (14.0 vs. 11.2 months; $p=0.00185$ ), radium-223 delayed the median time to first SRE by 5.2 months compared with placebo (13.6 vs. 8.4 months, $p=0.00046) .^{28}$ It should be noted, however, that since the full results of the phase III study have not been published, it is unknown whether the authors used the same definitions for SREs as was used in the studies by Fizazi ${ }^{24}$ and Saad. ${ }^{14}$

\section{Discussion}

\section{Choosing a bone-targeted therapy}

There are two bone-targeted therapies currently approved in Canada for use in CRPC patients with bone metastases. Choosing which therapy to use should be based on the advantages and disadvantages of each therapy, as well as the needs of each individual patient. As stated previously, IV zoledronic acid was the standard of care for CRPC patients with bone metastases, based on the study by Saad and colleagues that demonstrated a decreased proportion of SRES and a significant delay in median time to first SRE versus placebo. ${ }^{14}$ Recent evidence demonstrates that denosumab is superior to zoledronic acid in delaying the median time to the first on-study SRE by 3.6 months. ${ }^{24}$ The acute phase reactions and renal impairment monitoring associated with zoledronic acid are avoided with denosumab; however, the rate of hypocalcemia was higher for denosumab. Rates of drug-related adverse events in CRPC patients were similar for zoledronic acid and denosumab. Some additional considerations include route of administration/ease of use (SC for denosumab, IV for zoledronic acid), and reimbursement guidelines for each drug. Both zoledronic acid and denosumab have about the same acquisition costs; the difference in method of administration means a lower administration cost for denosumab. ${ }^{29} \mathrm{~A}$ full cost-comparison of these two agents is beyond the scope of this review. 
So et al.

\section{Initiating bone-targeted therapy}

Current clinical guidelines recommend that bone-targeted therapy should be initiated in CRPC patients who have evidence of bone metastases. ${ }^{30-32}$ The exact timing of initiating bone-targeted therapy is at the physician's discretion. Patients without bone pain have the same risk of developing SREs as those with bone pain; therefore early treatment with bone-targeted therapy could potentially prevent more SREs than waiting until a patient reports pain. ${ }^{26}$ The initiation of zoledronic acid prior to the onset of pain resulted in better outcomes (fewer SREs and longer time to onset of bone pain) compared with waiting for the onset of painful metastases. ${ }^{26}$ Bone scans may be conducted periodically for CRPC patients who do not yet have evidence of bone metastases, although there is no consensus on the frequency of these scans. The indication for a bone scan may be symptom-driven (e.g., bone pain) or driven by the patient's prostate-specific antigen level.

\section{Managing potential complications in patients receiving bone-targeted therapy}

Renal impairment, hypocalcemia, ONJ and acute phase reactions are the most common complications associated with bone-targeted therapies.

\section{Renal function}

A test for renal function, serum creatinine clearance, should be done before starting any bone-targeted therapy. Zoledronic acid is not recommended for patients with renal impairment (creatinine clearance of $<30 \mathrm{~mL} / \mathrm{min}$ ). ${ }^{30}$ During treatment, renal function should be monitored regularly to detect any signs of impairment, and the dose of zoledronic acid must be adjusted according to creatinine clearance obtained just prior to infusion. Denosumab is not dependent on the renal system for elimination; it is likely eliminated through the reticuloendothelial system similar to other therapeutic monoclonal antibodies. ${ }^{33}$ Therefore, denosumab is not contraindicated for patients with renal failure or on hemodialysis, although further study is required in patients with a creatinine clearance $<30 \mathrm{~mL} / \mathrm{min}^{30}$

Renal impairment associated with zoledronic acid can be managed by adjusting dosage at each administration to account for renal function, and infusion should be given in no less than 15 minutes. ${ }^{14}$

Acute phase reactions can also be associated with zoledronic acid. Symptoms include arthralgia, increased bone pain, low-grade fever, myalgia and nausea; these generally last 24 to 48 hours for the first or second infusions only. Non-steroidal anti-inflammatory drugs (NSAID) analgesics may be given as needed. ${ }^{34}$

\section{Hypocalcemia}

Hypocalcemia is an expected on-target effect and is a concern for both therapies $(16 \%$ vs. $8 \%$ for denosumab and zoledronic acid in patients with CRPC, respectively). ${ }^{24}$ Therefore, baseline calcium should be measured, and any baseline hypocalcemia must be resolved before beginning treatment. In clinical guidelines, monitoring serum calcium is required during therapy with denosumab and recommended with zoledronic acid; calcium supplementation is essential during either therapy. ${ }^{30}$ The minimum daily doses of calcium and vitamin D are $500 \mathrm{mg}$ and $400 \mathrm{IU}$, respectively. ${ }^{24}$ Moderate or severe hypocalcemia can be treated with supplementation as necessary. ${ }^{30}$

Since denosumab's approval for use in patients with CRPC and other forms of advanced cancer, the associated clinical rate of severe hypocalcemia has been $1 \%$ to $2 \%$, including some cases that were fatal. ${ }^{35} \mathrm{~A}$ further recommendation to minimize the risk of severe hypocalcemia is to identify those at highest risk, which include patients with renal impairment or on dialysis. It may be necessary to reassess the benefit of denosumab treatment in those patients who suffer from severe symptomatic hypocalcemia.

\section{Osteonecrosis of the jaw}

An oral examination and instruction in good oral hygiene are recommended when initiating bone-targeted therapy. Tooth extraction $(64.9 \%$ vs. $59.6 \%)$, jaw pain $(67.6 \%$ vs. $92.3 \%)$, and oral infection ( $45.9 \%$ vs. $50 \%$ ) were associated with cases of $\mathrm{ONJ}$ in prostate cancer, breast cancer, multiple myeloma and other cancer patients receiving zoledronic acid or denosumab, respectively. ${ }^{36}$ Patients should be monitored for any dental or oral pain, although most important is avoiding invasive dental procedure while on therapy to reduce the risk of $\mathrm{ONJ} .{ }^{32}$

Managing ONJ can be daunting for urologists and oncologists. Decisions regarding the management or prevention of ONJ may be made in consultation with a dentist or oral surgeon. In a combined analysis of three prospective trials comparing zoledronic acid and denosumab in patients with metastatic cancer, including prostate cancer, $23,24,36,37$ ONJ was infrequent $1.8 \%$ for denosumab and $1.3 \%$ for zoledronic acid) and conservative management resolved over one-third of the cases. ${ }^{36}$

Symptoms of ONJ include pain, swelling and infection of soft tissues, loosening of teeth, drainage, and a feeling of heaviness or numbness in the jaw. The American Association of Oral and Maxillofacial Surgeons (AAOMS) recommend a conservative approach to ONJ treatment through the use of antibiotics, oral rinses and limited debridement. ${ }^{38}$ Patients with more severe $\mathrm{ONJ}$ may require more aggressive treatment, including surgery and bone resection. ${ }^{36}$ Bone-targeted 
therapy should be discontinued until the ONJ is resolved, and then restarted depending on the risks and benefits of treatment for the individual patient. ${ }^{38}$

\section{Other considerations}

Although bone turnover markers (e.g., NTx, BALP) may provide information regarding disease progression in the bone, routine monitoring of these markers is not used in the clinical management of CRPC. Zoledronic acid and denosumab have been shown to increase and decrease the levels of markers of bone formation and resorption, respectively, ${ }^{14,24}$ and the levels of these markers correlate with disease progression and overall survival, particularly for urinary NTx. ${ }^{10}$

Patients who have experienced one SRE are at a higher risk for subsequent SREs; treatment with zoledronic acid or denosumab after the first SRE continues to provide clinical benefit by reducing the risk of subsequent SREs and other skeletal morbidities. ${ }^{39,24}$ Therefore, unless other complications arise, it would seem prudent to continue a bone-targeted therapy if an SRE has occurred.

\section{Switching from one bone-targeted therapy to another}

There are few data regarding the advantages or disadvantages of switching from one bone- targeted therapy to another. No studies exist that examine the outcomes of switching from denosumab to zoledronic acid. There are no phase III data evaluating the efficacy of denosumab in prostate cancer patients who previously received zoledronic acid. In a phase II study, patients with prostate cancer who showed no improvement in serum or urinary bone markers while on IV bisphosphonates (zoledronic acid, pamidronate or ibandronate) were switched to denosumab or remained on IV bisphosphonate. A greater proportion of patients who were given denosumab (22 of 32, 69\%) compared with those who remained on IV bisphosphonate (3 of 16, 19\%) showed decreased urinary NTx. ${ }^{20}$ Although the study was not sufficiently powered to examine the rate of SREs, there was a lower proportion of prostate cancer patients in the denosumab group (1 of 33 patients; 3\%) compared with the IV bisphosphonate group (3 of 16 patients; 19\%) who experienced an SRE during the study. ${ }^{20}$ These results indicate that switching to denosumab from an IV bisphosphonate is feasible (if such a situation were to occur), although further study is required to examine more patients and to observe the toxicity profile when switching from one drug to another. ${ }^{30,40}$

\section{Duration of bone-targeted therapy}

According to the National Comprehensive Cancer Network, the optimal treatment duration for either zoledronic acid or denosumab is uncertain. ${ }^{30}$ However, zoledronic acid has been shown to be safe in patients with metastatic CRPC for up to 24 months. ${ }^{8}$ The most recent study that compares zoledronic acid and denosumab was event- and not time-driven; the median duration of treatment was 11.9 months. Safety data are available for a concurrent phase III trial comparing denosumab to placebo in patients with high-risk prostate cancer, with the median treatment duration for denosumab being 19 months. ${ }^{33}$ Results from the 2-year open-label extension of patients with metastatic breast cancer have provided safety data for nearly 5 years of denosumab therapy. At the same dose used for patients with CRPC, no new safety signals were found over this time period, although a patient's risk of ONJ over this period should be considered. The risk of $\mathrm{ONJ}$ is cumulative and therefore increases with extended bone-targeted therapy. For denosumab, the cumulative incidence of $\mathrm{ONJ}$ in patients with advanced breast cancer who received denosumab for up to 5 years was $4.7 \%{ }^{40}$ It should be noted, however, that the benefit of preventing SREs outweighs the risk of ONJ for both therapies. ${ }^{36}$

\section{Conclusions}

Until recently, zoledronic acid was the only bone-targeted therapy available to reduce the risk of SREs in patients with metastatic CRPC in Canada. Based on the positive results of a pivotal phase III study, denosumab has recently been approved in Canada and presents another bone-targeted therapy option for patients with metastatic CRPC.

Competing interests: Dr. So is on advisory boards for Amgen and Novartis. Dr. Chin has acted on an advisory board or as a speaker for Amgen and Novartis. Dr. Fleshner has not declared any competing interests. Dr. Saad has received consultant and research and honoraria from Novartis and Amgen.

Acknowledgements: The authors wish to thank Miranda Tradewell, PhD, for providing assistance with literature search and medical writing and Matthew Culham, Six Degrees Medical Consulting, for editorial assistance. Expenses associated with the preparation and submission of this manuscript were supported by an unrestricted educational grant from Amgen Canada Inc. The authors did not receive honoraria from Amgen or any other source for the preparation of this manuscript.

This paper has been peer-reviewed.

\section{References}

1. Prostate Cancer Statistics at a glance. Canadian Cancer Society; 2011. hittp://www.cancer.ca. Accessed November 21, 2012.

2. Petrylak DP, Tangen $C M$, Hussain $M H$, et al. Docetaxel and estramustine compared with mitoxantrone and prednisone for advanced refractory prostate cancer. N Engl J Med 2004;351:1513-20. http:// dx.doi.org/10.1056/NEJMo0041318

3. Scher HI, Morris MJ, Kelly WK, et al. Prostate cancer clinical trial end points: "RECIST"ing a step backwards. Clin Cancer Res 2005;11:5223-32. 
So et al.

4. Tannock IF, de Wit R, Berry WR, et al. Docetaxel plus prednisone or mitoxantrone plus prednisone for advanced prostate cancer. N Engl J Med 2004;351:1502-12. http://dx.doi.org/10.1056/ NEJMoa040720

5. Ibrahim A, Scher N, Williams G, et al. Approval summary for zoledronic acid for treatment of multiple myeloma and cancer bone metastases. Clin Cancer Res 2003;9:2394-9.

6. Lipton A. Pathophysiology of bone metastases: how this knowledge may lead to therapeutic intervention. I Support Oncol 2004;2:205-13; discussion 213-4, 216-7, 219-20.

7. Coleman RE. Skeletal complications of malignancy. Cancer 1997;80(8 Suppl):1588-94. http://dx.doi. org/10.1002/(SICI) 1097-0142(19971015)80:8+<1588::AID-CNCR9>3.0.C0;2-G

8. Saad F, Gleason DM, Murray R, et al. Long-Term Efficacy of Zoledronic Acid for the Prevention of Skeletal Complications in Patients With Metastatic Hormone-Refractory Prostate Cancer. J Natl Cancer Inst 2004:96:879-82. http://dx.doi.org/10.1093/inci/dihl41

9. Tchekmedyian NS, Chen YM, Saad F. Disease progression increases the risk of skeletal-related events in patients with bone metastases from castration-resistant prostate cancer, lung cancer, or other solid tumors. Cancer Invest 2010;28:849-55. http://dx.doi.org/10.3109/07357907.2010.483508

10. Brown JE, Cook RJ, Major P, et al. Bone turnover markers as predictors of skeletal complications in prostate cancer, lung cancer, and other solid tumors. J Natl Cancer Inst 2005;97:59-69. hittp://dx.doi. org/10.1093/inci/dij002

11. Fohr B, Dunstan CR, Seibel MJ. Clinical review 165: Markers of bone remodeling in metastatic bone disease. J Clin Endocrinol Metab 2003;88:5059-75. http://dx.doi.org/10.1210/ic.2003-030910

12. Weinfurt KP, L L Y , Castel LD, et al. The significance of skeletal-related events for the health- related quality of life of patients with metastatic prostate cancer. Ann Oncol 2005; 16:579-84.

13. Krupski TL, Foley KA, Baser 0 , et al. Health care cost associated with prostate cancer, androgen deprivation therapy and bone complications. J Urol 2007;178(4 Pt 1):1423-8. http://dx.doi.org/10.1016/i. juro.2007.05.135

14. Saad F, Gleason DM, Murray R, et al. A randomized, placebo-controlled trial of zoledronic acid in patients with hormone-refractory metastatic prostate carcinoma. J Natl Cancer Inst 2002;94:1458-68. http:// dx.doi.org/10.1093/inci/94.19.1458

15. Bogardus ST Jr, Towle V, Williams CS, et al. What does the medical record reveal about functional status? A comparison of medical record and interview data. J Gen Intern Med 2001;16:728-36. http://dx.doi. org/10.1111/.1.1525-1497.2001.00625.x

16. Saad F, Lipton A, Cook R, et al. Pathologic fractures correlate with reduced survival in patients with malignant bone disease. Cancer 2007;1 10:1860-7. http://dx.doi.org/10.1002/cncr.22991

17. Oefelein MG, Ricchiuti V, Conrad W, et al. Skelefal fractures negatively correlate with overall survival in men with prostate cancer. J Urol 2002;168:1005-7. http://dx.doi.org/10.1016/S0022-5347(05)64561-2

18. Braithwaite RS, Col NF, Wong JB. Estimating hip fracture morbidity, mortality and costs. J Am Geriatr Soc 2003:51:364-70. http://dx.doi.org/10.1046/i.1532-5415.2003.51110.x

19. O'Neill TW, Roy DK. How many people develop fractures with what outcome? Best practice \& research. Clin Rheum 2005;19:879-95. http://dx.doi.org/10.1016/i.berh.2005.06.003

20. Fizazi K, Bosserman L, Gao G, et al. Denosumab treatment of prostate cancer with bone metastases and increased urine $\mathrm{N}$-telopeptide levels after therapy with intravenous bisphosphonates: results of a randomized phase II trial. J Urol 2009;182:509-15; discussion 515-6.

21. Dranitsaris $G$, Hsu T. Cost utility analysis of prophylactic pamidronate for the prevention of skeletal related events in patients with advanced breast cancer. Support Care Cancer 1999;7:271-9.

22. Health Canada. Notice of Compliance for denosumab (XGeva) for advanced prostate cancer 2011.

23. Stopeck AT, Lipton A, Body JJ, et al. Denosumab compared with zoledronic acid for the treatment of bone metastases in patients with advanced breast cancer: a randomized, double- blind study. J Clin Oncol 2010;28:5132-9.

24. Fizazi K, Carducci M, Smith M, et al. Denosumab versus zoledronic acid for treatment of bone metastases in men with castration-resistant prostate cancer: a randomised, double-blind study. Lancet 2011;377:81322. http://dx.doi.org/10.1016/S0140-6736(10)62344-6

25. Health Canada. Notices of Compliance, January 1-December 31, 2002. 2002. hittp://www.hc-sc.gc.ca/ dhp-mps/alt_formats/hpfb-dgpsa/txt/prodpharma/pre2002et.txt. Accessed November 26, 2012.

26. Saad F, Eastham J. Zoledronic Acid improves clinical outcomes when administered before onset of bone pain in patients with prostate cancer. Urology 2010;76:1175-81. http://dx.doi.org/10.1016/i.urology.2010.05.026
27. Suominen MI, Rissanen JP, Käkönen R, et al. Alpharadin inhibits osteoclast differentiation in vitro and progression of established breast cancer bone metastases in vivo. In: Proceedings of the 102nd Annual Meeting of the American Association for Cancer Research; 2011 Apr 2-6; Orlando, Florida. Philadelphia (PA): AACR; 2011. Abstract 2664.

28. Parker C, Heinrich D, O'Sullivan JM, et al. Overall Survivial Benefit of Radium-223 Chloride (AlpharadinTM) in the Treatment of Patients with Symptomatic Bone Metastases in Castration-Resistant Prostate CAncer (CRPC): A Phase III Randomised Trial (ALSYMPCA). In: Proceedings of the European Society of Medical Oncology; 2011 May 16-19; Providence (RI).

29. Health Canada. CDEC Final Recommendation, Denosumab (Xgeva - Amgen Canada Inc). Common Drug Review. 2011:1-5.

30. Network NCC. NCCN Clinical Practice Guidelines in Oncology 2011. http://www.nccn.org/professionals/ physician_gls/f_guidelines.asp. Accessed November 26, 2012.

31. Heidenreich A, Bolla M, Joniau S, et al. Guidelines on Prostate Cancer. 2011. http://www.uroweb.org/ gls/pdf/08_Prostate_Cancer\%20September\%2022nd\%202011.pdf. Accessed November 26, 2012.

32. Saad F, Hotte S. Guidelines for the management of castrate-resistant prostate cancer. CUA Guideline. Can Urol Assoc J 2010;4:380-4.

33. Smith MR, Saad F, Coleman R, et al. Denosumab and bone-metastasis-free survival in men with castrationresistant prostate cancer: results of a phase 3, randomised, placebo-controlled trial. Lancet 2012;379:3946. Epub 2011 Nov 15.

34. Polascik TJ. Bone health in prostate cancer patients receiving androgen-deprivation therapy: the role of bisphosphonates. Prostate Cancer Prostatic Dis 2008;11:13-9. http://dx.doi.org/10.1038/ si.pcan. 4501019

35. Health Canada. Health Canada Endorsed Important Safety Information on XGEVA(R) (denosumab). http://hc-sc.gc.ca/dhp-mps/medeff/advisories-avis/prof/_2012/xgeva_hpc-cps-eng.php. (Accessed November 26, 2012)

36. Saad F, Brown JE, Van Poznak C, et al. Incidence, risk factors, and outcomes of osteonecrosis of the jaw: integrated analysis from three blinded active-controlled phase III trials in cancer patients with bone metastases. Ann Oncol 2012;23:1341-7. Epub 2011 Oct 10.

37. Henry DH, Costa L, Goldwasser F, et al. Randomized, double-blind study of denosumab versus zoledronic acid in the treatment of bone metastases in patients with advanced cancer (excluding breast and prostate cancer) or multiple myeloma. J Clin Oncol 2011;29:1125-32.

38. Advisory Task Force on Bisphosphonate-Related Ostenonecrosis of the Jaws, American Association of Oral and Maxillofacial Surgeons. American Association of Oral and Maxillofacial Surgeons position paper on bisphosphonate-related osteonecrosis of the jaws. J Oral Maxillofac Surg 2007;65:369-76.

39. Saad F, Chen YM, Gleason DM, et al. Continuing benefit of zoledronic acid in preventing skeletal complications in patients with bone metastases. Clin Genitourin Cancer 2007;5:390-6. hittp://dx.doi. org/10.3816/CGC.2007.n.022

40. Stopeck AT, Lipton A, Martin M, et al. Denosumab in Patients With Breast Cancer and Bone Metastases Previously Treated With Zoledronic Acid or Denosumab: Results From the 2-Year Open-Label Extension Treatment Phase of a Pivotal Phase 3 Study. In: Proceedings from the San Antonio Breast Cancer Symposium 2011.

41. Dearnaley DP, Sydes MR, Mason MD, et al. A double-blind, placebo-controlled, randomized trial of oral sodium clodronate for metastatic prostate cancer (MRC PR05 Trial). J Natl Cancer Inst 2003;95:1300-11. http://dx.doi.org/10.1093/inci/dig038

42. Small EJ, Smith MR, Seaman JJ, et al. Combined analysis of two multicenter, randomized, placebocontrolled studies of pamidronate disodium for the palliation of bone pain in men with metastatic prostate cancer. J Clin Oncol 2003:21:4277-84.

Correspondence: Dr. Fred Saad, Director of Urologic Oncology, Centre Hospitalier de l'Université de Montréal, CHUM - Pavillon Notre-Dame, 1560 Sherbrooke East, Department of Urology, Montréal, QC H2L 4M1; fax: 514-412.7620; fredsaad@videotron.ca 